\title{
Changes in Aerobic Capacity Over Time in Elderly Patients With Acute Myocardial Infarction During Cardiac Rehabilitation
}

\author{
Ki-Hong Kim, MD, Yun-Chol Jang, MD, Min-Keun Song, MD, Hyeng-Kyu Park, MD, PhD, \\ In-Sung Choi, MD, PhD, Jae-Young Han, MD, PhD
}

Department of Physical and Rehabilitation Medicine, Chonnam National University Medical School and Hospital, Gwangju, Korea

Objective To test the hypothesis that a longer duration of phase II cardiac rehabilitation is required to recover the exercise capacity of elderly patients compared to younger patients.

Methods We retrospectively reviewed and analyzed the medical records of patients who were referred to our cardiac rehabilitation (CR) center and underwent percutaneous coronary intervention for acute myocardial infarction (AMI). A total of 70 patients were enrolled who underwent an exercise tolerance test (ETT) 3 weeks after the occurrence of an AMI (T0), 6 weeks after the first ETT (T1), and 12 weeks after the first ETT (T2). Patients older than 65 years were assigned to the elderly group $(n=24)$ and those aged 65 years and younger to the younger group $(\mathrm{n}=46)$. Both groups performed center-based or home-based CR for 12 weeks (3 times per week and 1 session per day). Exercise intensity for each individual was based on the target heart rate calculated by the Karvonen formula. The change in maximal metabolic equivalents $\left(\mathrm{MET}_{\max }\right)$ of the two groups was measured at each assessment point (T0, T1, and T2) to investigate the recovery of exercise capacity.

Results The younger group showed improvement in $\mathrm{MET}_{\max }$ between $\mathrm{T} 0$ and $\mathrm{T} 1$. However, $\mathrm{MET}_{\max }$ of the elderly group showed no significant improvement between $\mathrm{T} 0$ and $\mathrm{T} 1$. The exercise capacity, measured with $\mathrm{MET}_{\max }$, of all groups showed improvement between T0 and T2.

Conclusion Elderly patients with AMI need a longer duration of CR ( $>6$ weeks) than younger patients with AMI.

Keywords Cardiac rehabilitation, Old age, Acute myocardial infarction, Exercise capacity, Exercise tolerance test

Received April 15, 2019; Accepted July 25, 2019

Corresponding author: Jae-Young Han

Department of Physical and Rehabilitation Medicine, Chonnam National University Hospital, 42 Jebong-ro, Dong-gu, Gwangju 61469, Korea. Tel: +8262-220-5198, Fax: +82-62-228-5975, E-mail: rmhanjy@daum.net

ORCID: Ki-Hong Kim (https://orcid.org/0000-0003-0827-6813); Yun-Chol Jang (https://orcid.org/0000-0001-5145-5062); Min-Keun Song (https:// orcid.org/0000-0001-8186-5345); Hyeng-Kyu Park (https://orcid.org/0000-0003-3769-6227); In-Sung Choi (https://orcid.org/0000-0003-3967-9201); Jae-Young Han (https://orcid.org/0000-0003-1672-8875).

() This is an open-access article distributed under the terms of the Creative Commons Attribution Non-Commercial License (http://creativecommons.org/ licenses/by-nc/4.0) which permits unrestricted noncommercial use, distribution, and reproduction in any medium, provided the original work is properly cited. Copyright $\odot 2020$ by Korean Academy of Rehabilitation Medicine 


\section{INTRODUCTION}

Cardiac rehabilitation (CR) is known to improve the exercise capacity and health-related quality of life of patients, thereby reducing risk factors and mortality rates associated with cardiac diseases, such as acute myocardial infarction (AMI) [1].

According to randomized clinical trials and metaanalyzes, CR has been shown to effectively promote functional recovery and improve exercise tolerance $[2,3]$. It is known that exercise tolerance can be improved through participation in CR and is also effective in elderly patients with high or very high cardiovascular risk $[4,5]$. Additionally, exercise-based CR programs were associated with improvement in physical performance such as peak oxygen consumption and distance walked in a 6-minute walk test. This was true even in older adults (aged 75 and older) who experienced an acute coronary event or had cardiac surgical intervention, particularly in those with poorer baseline performance [6].

CR is composed of three major periods: phase I, phase II, and phase III. Among them, phase II CR begins after a symptom-limited full level exercise tolerance test (ETT) for patients with cardiac disease. This phase consists of an outpatient training phase with secondary prevention, intense education, and aerobic conditioning [7].

However, the duration of phase II CR varies per study [8]. For example, in a recent review of CR guidelines, the recommended duration ranged from a minimum of 3 weeks in Germany to a maximum of 12 months in Austria [9]. Furthermore, the duration of phase II CR in Europe is between 3 to 14 weeks and averages 8 weeks. When outpatient rehabilitation was performed, the duration of phase II programs ranged from 7 to 26 weeks [10].

What duration of CR is needed to be effective? Cardiac rehabilitation studies with more than two sessions per week for multiple weeks (from 2 to 28 weeks) improved the exercise capacity of patients with coronary artery disease [11]. The World Health Organization recommends phase II CR lasting 6 to 8+ weeks [9]. As seen in the literature, the optimal duration of phase II CR to improve aerobic capacity will vary depending on the patient's condition, and the basis for optimal duration is still lacking. There is also a lack of data for elderly AMI patients.

The purpose of this study is to test the hypothesis that a longer duration of phase II CR is required to recover the exercise capacity of elderly patients compared to younger patients.

\section{MATERIALS AND METHODS}

We retrospectively reviewed and analyzed the medical records of 286 patients, who were referred to our cardiac rehabilitation center after percutaneous coronary intervention, with AMI such as ST-elevation myocardial infarction (STEMI) and non-ST-elevation myocardial infarction (NSTEMI) between January 2011 and September 2017. Patients who underwent ETT three times at specific times were enrolled. Specific ETT times are defined as follows: 3 weeks after the occurrence of AMI (T0), 6 weeks after of the first ETT (T1, the time range is \pm 3 weeks), and 12 weeks after the first ETT (T2, the time range is \pm 6 weeks). ETT was performed with symptomlimited, modified Bruce protocol.

The following patients were excluded: (1) patients with recurrence of AMI or re-admission during phase II CR and patients with musculoskeletal problems such as paralysis (lesser than grade 3 in the manual muscle test) or severe pain (7 or higher in the visual analog scale); (2) patients with low risk factors on the risk classification (first ETT $\geq 7 \mathrm{MET}_{\max }$ or left ventricle ejection fraction $[\mathrm{LVEF}]<50 \%$ ); and (3) patients who did not undergo ETT at $\mathrm{T} 0, \mathrm{~T} 1$, or $\mathrm{T} 2$. The time ranges of $\mathrm{T} 1$ were \pm 3 weeks and the time ranges of $\mathrm{T} 2$ were \pm 6 weeks; patients who underwent ETT outside these time ranges were excluded. Ultimately, 70 patients (54 men, 16 women) were included in this study. Patients older than 65 years were assigned to the elderly group $(n=24)$ and the remaining patients were assigned to the younger group $(n=46)$. This study was approved by the Chonnam National University Hospital Institutional Review Board (No. CNUH-2018-087). All patients chose to perform center-based or home-based phase II CR for 12 weeks (T0 to T2). The CR program was 3 days per week with one session per day at the CR center or the patient's home. A CR session consisted of a 10-minute warm-up exercise, 30 minutes of aerobic exercise, and 10 minutes for a cool-down exercise. The aerobic exercise intensity was based on the individual's target heart rate (HR) calculated by the Karvonen formula [(maximal HR - resting HR) $\times$ training\% + resting HR] after ETT. We prescribed exercise intensity at $55 \%-70 \%$ for patients with a moderate risk and at $40 \%-55 \%$ for those 
with a severe risk. Patient who underwent home-based CR programs were checked by self-monitoring diaries, phone calls from staff, or exercise history records.

Study outcomes, such as exercise capacity (measured using $\mathrm{MET}_{\max }$ ), resting and maximal HR, resting systolic blood pressure (SBP), and total exercise time (TET), were measured at T0, T1, and T2. Resting HR and resting SBP were checked before ETT. MET $_{\max }$, maximal HR, and TET were checked during ETT. Before the ETT was performed, the underlying diseases, medication history, type of AMI, LVEF, and body mass index (BMI) were recorded.

Data were statistically analyzed with IBM SPSS Statistics version 19.0 (IBM Corp., Armonk, NY, USA). We used the paired t-test, independent t-test, and chi-square test to compare categorical variables including $\mathrm{MET}_{\max }$, underlying diseases, medication history, type of AMI, LVEF, BMI, and more. For all statistical tests, a p-value less than 0.05 was considered significant. The changing ratio was also used in the analysis. The changing ratio is a statistical method that can be used to compare two groups with different initial values. The calculation method of chang- ing ratio is as follows. For example, the changing ratio of $\mathrm{MET}_{\text {max }}$ (T0 to T1) is $\frac{\mathrm{MET}_{\text {max }}(\mathrm{T} 1)-\mathrm{MET}_{\text {max }}(\mathrm{T} 0)}{\mathrm{MET}_{\text {max }}(\mathrm{T} 0)} \times 100$.

\section{RESULTS}

\section{Clinical characteristics of the study population}

The clinical characteristics of the study population, including age, sex, LVEF, BMI, medication (such as betablocker usage), presence of hypertension, dyslipidemia, and diabetes mellitus, and coronary heart disease (CHD) types, are shown in Table 1. The mean age of all patients was 60.1 years (elderly group, 72.8 years; younger group, 53.5 years). The maximum and minimum ages of the elderly group were 82 and 65 years, respectively, and those of the younger group were 63 and 37 years, respectively. The LVEF of all patients was $56.00 \%$ and that of the elderly and younger groups was $60.97 \%$ and $53.41 \%$, respectively. When both groups were analyzed by the chi-square test, the types of CHD and medication, such as beta-blockers, angiotensin-converting enzyme inhibitors, and statins, were similar between the elderly and

Table 1. Clinical characteristics of the study population

\begin{tabular}{|c|c|c|c|c|}
\hline & Whole group $(\mathrm{n}=70)$ & Elderly group $(n=24)$ & Younger group $(n=46)$ & p-value \\
\hline Age (yr) & $60.1 \pm 11.0$ & $72.8 \pm 5.1$ & $53.5 \pm 6.6$ & $<0.001^{*}$ \\
\hline Sex, male & $53(75.7)$ & $14(70.8)$ & $39(84.7)$ & $0.014^{*}$ \\
\hline $\operatorname{BMI}\left(\mathrm{kg} / \mathrm{m}^{2}\right)$ & $24.68 \pm 3.38$ & $23.00 \pm 3.27$ & $25.55 \pm 3.12$ & $0.003^{*}$ \\
\hline $\operatorname{LVEF}(\%)$ & $56.00 \pm 9.55$ & $60.97 \pm 7.87$ & $53.41 \pm 9.39$ & $0.001^{*}$ \\
\hline Hypertension & $32(45.7)$ & $15(62.5)$ & 17 (36.9) & $0.041^{*}$ \\
\hline Dyslipidemia & $22(31.4)$ & $5(20.8)$ & $17(36.9)$ & 0.167 \\
\hline Diabetes mellitus & $20(28.5)$ & $7(29.1)$ & $13(28.2)$ & 0.936 \\
\hline CHD type & & & & 0.507 \\
\hline STEMI & $37(52.9)$ & $14(58.3)$ & $23(50.0)$ & \\
\hline NSTEMI & $33(47.1)$ & $10(41.6)$ & $23(50.0)$ & \\
\hline CR type & & & & 0.157 \\
\hline Center-based CR & $12(17.1)$ & $2(8.3)$ & $10(21.7)$ & \\
\hline Home-based CR & $58(82.9)$ & $22(91.7)$ & $36(79.3)$ & \\
\hline \multicolumn{5}{|l|}{ Medication } \\
\hline B-blocker & $50(71.4)$ & $17(70.8)$ & $33(71.7)$ & 0.936 \\
\hline ACEI & $30(42.8)$ & $8(33.3)$ & $22(47.8)$ & 0.244 \\
\hline Statin & $36(51.4)$ & $12(50.0)$ & $24(52.1)$ & 0.862 \\
\hline
\end{tabular}

Values are presented as mean \pm standard deviation or number (\%).

BMI, body mass index; LVEF, left ventricular ejection fraction; CHD, coronary heart disease; STEMI, ST elevation myocardial infarction; NSTEMI, non-ST elevation myocardial infarction; CR, cardiac rehabilitation; ACEI, angiotensinconverting enzyme inhibitor.

${ }^{*} \mathrm{p}<0.05$, in comparison of the Elderly and Younger groups. 


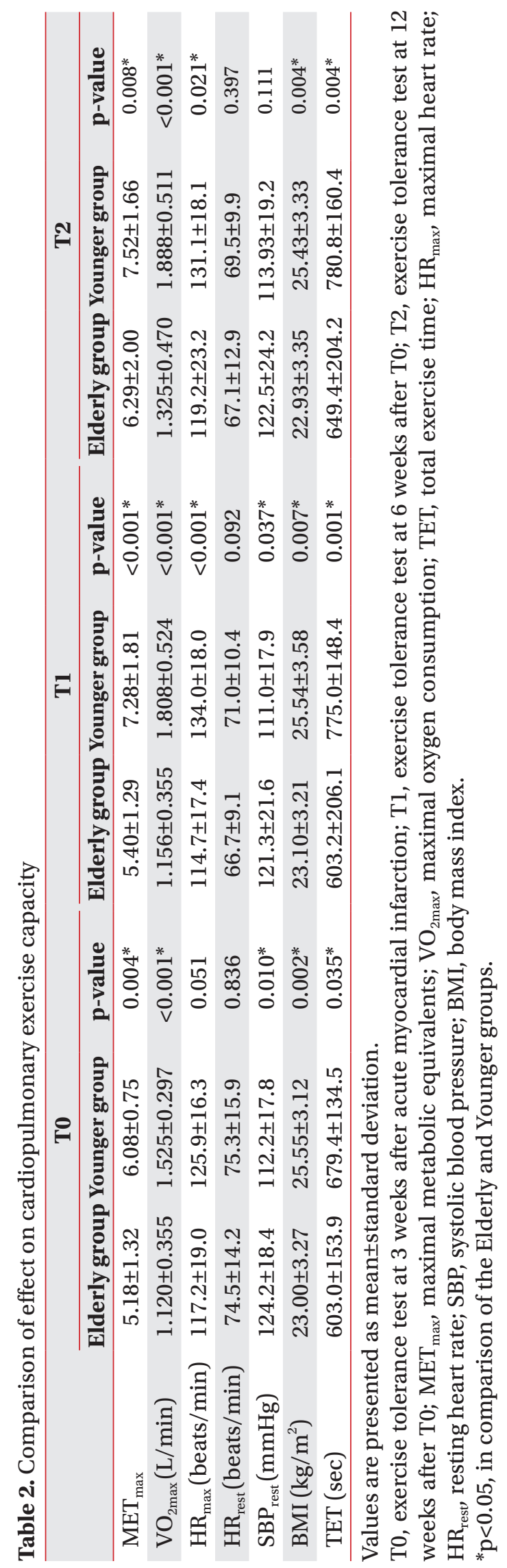

younger groups $(\mathrm{p}=0.507, \mathrm{p}=0.936, \mathrm{p}=0.244$, and $\mathrm{p}=0.862$, respectively). Additionally, the types of CR were similar between the elderly and younger groups $(p=0.157)$. In the elderly group, 2 patients participated in the center-based $\mathrm{CR}$, while the others participated in home-based CR. In the younger group, 10 patients participated in the centerbased CR and 36 patients participated in home-based $\mathrm{CR}$. The younger group had more male patients than the elderly group $(\mathrm{p}=0.014)$. BMI $(\mathrm{p}=0.003)$ in the elderly group and LVEF $(\mathrm{p}=0.001)$ in the younger group were relatively low. However, there was no difference between the two groups in underlying disease except hyperten$\operatorname{sion}(\mathrm{p}=0.041)$.

\section{Comparison of effects on cardiopulmonary exercise capacity}

We compared the effects of cardiopulmonary exercise capacity in each period, at $\mathrm{T} 0, \mathrm{~T} 1$, and $\mathrm{T} 2$. The younger group showed higher values of $\mathrm{MET}_{\max }$ and TET at T0, $\mathrm{T} 1$, and $\mathrm{T} 2$ than the elderly group. At T0, $\mathrm{MET}_{\max }$ of the younger and elderly groups was $6.08 \pm 0.75$ and $5.18 \pm 1.32$, respectively ( $\mathrm{p}=0.004)$, and TETs were $679.4 \pm 134.5$ and $603.0 \pm 153.9$, respectively ( $\mathrm{p}=0.035)$. At $\mathrm{T} 1, \mathrm{MET}_{\max }$ and TET of the younger and elderly groups was $7.28 \pm 1.81$ and $5.40 \pm 1.29(\mathrm{p}<0.001)$ and $775.0 \pm 148.4$ and 603.2 \pm 206.1 $(\mathrm{p}=0.001)$, respectively. At T2, $\mathrm{MET}_{\max }$ and TET of the younger and elderly groups was $7.52 \pm 1.66$ and $6.29 \pm 2.00$ $(\mathrm{p}=0.008)$ and $780.8 \pm 160.4$ and $649.4 \pm 204.2(\mathrm{p}=0.004)$, respectively (Table 2).

The younger group showed improvement of $\mathrm{MET}_{\max }$ between T0 and T1. Baseline $\mathrm{MET}_{\max }$ of the younger group was $6.08 \pm 0.75$ and $7.28 \pm 1.81$ after 6 weeks $(\mathrm{p}<0.001)$ (Table 3 ). However, the elderly group showed no significant improvement of $\mathrm{MET}_{\max }$ between $\mathrm{T} 0$ and $\mathrm{T} 1$. Baseline $\mathrm{MET}_{\text {max }}$ of the elderly group was $5.18 \pm 1.32$ and $5.40 \pm 1.29$ after 6 weeks ( $\mathrm{p}=0.455)$ (Table 3$)$.

The exercise capacity ( $\mathrm{MET}_{\max }$ ) of both groups showed improvement between T0 and T2. Baseline $\mathrm{MET}_{\max }$ of the younger group was $6.08 \pm 0.75$ and $7.52 \pm 1.66$ after 12 weeks. Baseline $\mathrm{MET}_{\max }$ of the elderly group was $5.18 \pm 1.32$ and $6.29 \pm 2.00$ after 12 weeks (younger group, $\mathrm{p}=0.001$; elderly group, $\mathrm{p}=0.031$ ) (Table 3 ).

The changing ratio of $\mathrm{MET}_{\max }$ in the elderly and younger groups

The changing ratio was used to correct the initial values 
Table 3. Cardiopulmonary exercise capacity at $\mathrm{T} 0, \mathrm{~T} 1$, and $\mathrm{T} 2$

\begin{tabular}{|c|c|c|c|c|c|}
\hline & \multirow{2}{*}{ To } & \multirow{2}{*}{$\mathbf{T 1}$} & \multirow{2}{*}{ T2 } & \multicolumn{2}{|c|}{ p-value } \\
\hline & & & & T0-T1 & T0-T2 \\
\hline \multicolumn{6}{|l|}{ Elderly group } \\
\hline $\mathrm{MET}_{\max }$ & $5.18 \pm 1.32$ & $5.40 \pm 1.29$ & $6.29 \pm 2.00$ & 0.455 & $0.031^{*}$ \\
\hline $\mathrm{VO}_{2 \max }(\mathrm{L} / \mathrm{min})$ & $1.120 \pm 0.355$ & $1.156 \pm 0.355$ & $1.325 \pm 0.470$ & 0.542 & $0.046^{*}$ \\
\hline $\mathrm{HR}_{\max }$ (beats/min) & $117.2 \pm 19.0$ & $114.7 \pm 17.4$ & $119.2 \pm 23.2$ & 0.495 & 0.604 \\
\hline $\mathrm{HR}_{\text {rest }}$ (beats/min) & $74.5 \pm 14.2$ & $66.7 \pm 9.1$ & $67.1 \pm 12.9$ & $0.009^{*}$ & $0.032^{*}$ \\
\hline $\mathrm{SBP}_{\text {rest }}(\mathrm{mmHg})$ & $124.2 \pm 18.4$ & $121.3 \pm 21.6$ & $122.5 \pm 24.2$ & 0.491 & 0.718 \\
\hline BMI $\left(\mathrm{kg} / \mathrm{m}^{2}\right)$ & $23.00 \pm 3.27$ & $23.10 \pm 3.21$ & $22.93 \pm 3.35$ & 0.296 & 0.836 \\
\hline TET (sec) & $603.0 \pm 153.9$ & $603.2 \pm 206.1$ & $649.4 \pm 204.2$ & 0.997 & 0.24 \\
\hline \multicolumn{6}{|l|}{ Younger group } \\
\hline $\mathrm{MET}_{\max }$ & $6.08 \pm 0.75$ & $7.28 \pm 1.81$ & $7.52 \pm 1.66$ & $<0.001^{*}$ & $<0.001^{*}$ \\
\hline $\mathrm{VO}_{2 \max }(\mathrm{L} / \mathrm{min})$ & $1.525 \pm 0.297$ & $1.808 \pm 0.524$ & $1.888 \pm 0.511$ & $<0.001^{*}$ & $<0.001^{*}$ \\
\hline $\mathrm{HR}_{\max }$ (beats/min) & $125.9 \pm 16.3$ & $134.0 \pm 18.0$ & $131.1 \pm 18.1$ & $0.001^{*}$ & $0.029^{*}$ \\
\hline $\mathrm{HR}_{\text {rest }}($ beats/min) & $75.3 \pm 15.9$ & $71.0 \pm 10.4$ & $69.5 \pm 9.9$ & $0.021^{*}$ & $0.005^{*}$ \\
\hline $\mathrm{SBP}_{\text {rest }}(\mathrm{mmHg})$ & $112.2 \pm 17.8$ & $111.0 \pm 17.9$ & $113.93 \pm 19.2$ & 0.615 & 0.549 \\
\hline $\operatorname{BMI}\left(\mathrm{kg} / \mathrm{m}^{2}\right)$ & $25.55 \pm 3.12$ & $25.54 \pm 3.58$ & $25.43 \pm 3.33$ & 0.965 & 0.685 \\
\hline TET (sec) & $679.4 \pm 134.5$ & $775.0 \pm 148.4$ & $780.8 \pm 160.4$ & $0.001^{*}$ & $<0.001^{*}$ \\
\hline
\end{tabular}

Values are presented as mean \pm standard deviation.

T0, exercise tolerance test at 3 weeks after acute myocardial infarction; T1, exercise tolerance test at 6 weeks after T0; $\mathrm{T} 2$, exercise tolerance test at 12 weeks after T0; $\mathrm{MET}_{\max }$, maximal metabolic equivalents; $\mathrm{VO}_{2 \max }$, maximal oxygen consumption; TET, total exercise time; $\mathrm{HR}_{\max }$, maximal heart rate; $\mathrm{HR}_{\text {rest }}$, resting heart rate; $\mathrm{SBP}$, systolic blood pressure; BMI, body mass index.

*p $<0.05$, in comparison of the $\mathrm{T} 0$, and $\mathrm{T} 1, \mathrm{~T} 2$.

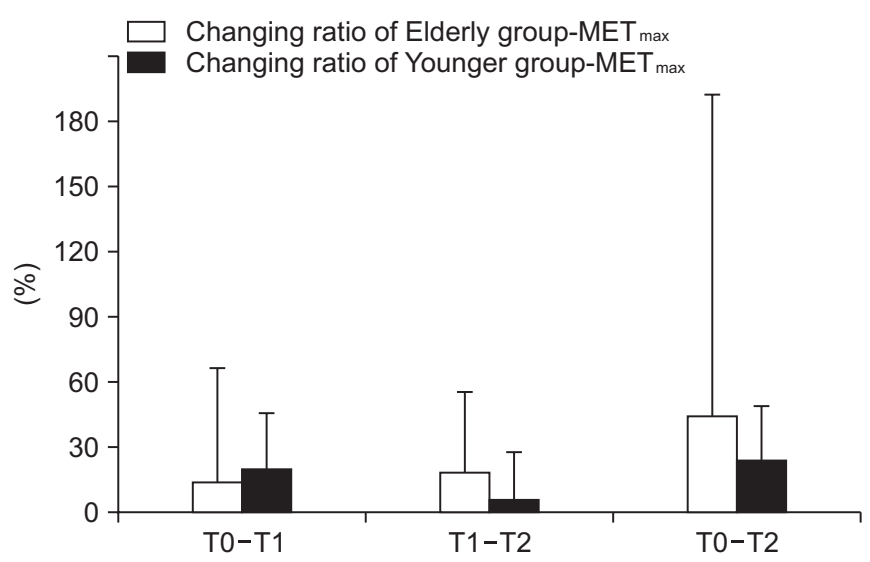

Fig. 1. Changing ratio of cardiopulmonary exercise capacity at T0, T1, and T2. Values are presented as mean \pm standard deviation. T0, exercise tolerance test at 3 weeks after acute myocardial infarction; T1, exercise tolerance test at 6 weeks after T0; T2, exercise tolerance test at 12 weeks after T0; $\mathrm{MET}_{\max }$, maximal metabolic equivalents. Changing ratio of $\mathrm{MET}_{\max }(\mathrm{T} 0-\mathrm{T} 1)$ is $\frac{\mathrm{MET}_{\max }(\mathrm{T} 1)-\mathrm{MET}_{\max }(\mathrm{T} 0)}{\mathrm{MET}_{\max }(\mathrm{T} 0)} \times 100$. of both groups. The changing ratios of the elderly and younger group in $\mathrm{MET}_{\max }$ were as follows. Between T0 and $\mathrm{T} 1$, the $\mathrm{MET}_{\max }$ changing ratio of the elderly group and younger group was $13.72 \%$ and $19.84 \%$, respectively. The changing ratio of the younger group was relatively larger than the elderly group. However, between $\mathrm{Tl}$ and $\mathrm{T} 2$, the $\mathrm{MET}_{\max }$ changing ratio of the elderly group was $18.22 \%$ and of the younger group was $5.54 \%$. Unlike T0$\mathrm{T} 1$, changing ratio of elderly group was relatively larger than younger group (Fig. 1).

\section{DISCUSSION}

McConnell et al. [1] compared changes in exercise capacity with age and found that maximal exercise capacity decreased with age, but the improvements achieved by CR were similar at all ages. According to Lavie et al. [2], elderly patients (65 years and older) with cardiovascular disease who enrolled in CR had lower baseline exercise capacity but had significant improvements after CR. In 
this study, the duration of CR was 12 weeks. Lavie and Milani [4] also showed the beneficial effects of CR and exercise training in the elderly, including modest improvements in lipids, obesity indexes, behavioral characteristics, and quality-of-life parameters, and marked improvements in exercise capacity.

The age related study from Lavie and Milani [12] showed elderly patients had a lower estimated aerobic capacity $(-27 \% ; \mathrm{p}<0.001)$ and directly measured peak oxygen consumption $\left(\mathrm{VO}_{2}\right)(-19 \% ; \mathrm{p}<0.01)$ before the initiation of CR. After CR, patients in the elderly cohort demonstrated significant improvements in estimated aerobic capacity $(+32 \%$; $<<0.0001)$ and peak $\mathrm{VO}_{2}(+13 \%$; $\mathrm{p}<0.0001)$. According to Menezes et al. [13], elderly patients had a lower estimated aerobic capacity and directly measured $\mathrm{VO}_{2 \max }$. However, following $\mathrm{CR}$, patients in the elderly cohort demonstrated significant improvements in estimated aerobic capacity and $\mathrm{VO}_{2 \max }$. The review by Pasquali et al. [14] showed that, at baseline, elderly patients are significantly less fit, having lower measured $\left(\mathrm{VO}_{2 \max },-19 \%\right.$ to $\left.-30 \%\right)$ and estimated $\left(\mathrm{MET}_{\max },-27 \%\right.$ to $-42 \%$ ) exercise capacity than younger patients. Despite lower initial values, studies have shown significant improvements in $\mathrm{VO}_{2 \max }(+13 \%$ to $+27 \%)$ and estimated exercise capacity $\left(\mathrm{MET}_{\max },+32 \%\right.$ to $\left.+43 \%\right)$ in elderly patients after CR.

In this study, we placed patients older than 65 years into the elderly group and the others were placed in the younger group. The clinical characteristics of both groups showed little differences. The younger group had more male patients than the elderly group $(\mathrm{p}=0.014)$. BMI $(p=0.003)$ in the elderly group and LVEF $(p=0.001)$ in the younger group were relatively low. CR effects did not show much difference between men and women [15]. LVEF ( $p=0.001$ ) in the younger group was relatively low compared to the elderly group. Excluding the low risk patients, it is estimated that more patients with low ejection fraction were included.

We performed center-based or home-based CR for 12 weeks according to the patient's choice. Both groups participated in CR three times a week and once per day. One session consisted of a warm-up exercise (10 minutes), aerobic exercise (30 minutes), and a cool-down exercise (10 minutes). The aerobic exercise intensity for each individual was based on the target HR calculated by the Karvonen formula after ETT. Patients who selected center- based CR participated from 6 weeks (short participation) to 12 weeks. On average, the frequency of center-based CR was $1.6 \pm 0.8$ times per week. On the days CR was not performed at the center, home-based CR was performed in parallel.

According to Anderson et al. [16], there is no significant difference in exercise capacity outcomes for patients receiving home-based or center-based CR in the shortterm (3-12 months). In this review, home-based CR is defined as a structured program with clear objectives for the participants, including monitoring, follow-up visits, and letters or phone calls from staff or self-monitoring diaries. In this study, patients who underwent homebased CR programs were checked by self-monitoring diaries, phone calls from staff, or exercise history recording.

Our study showed that $\mathrm{MET}_{\text {max }}$ of the younger group improved between $\mathrm{T} 0$ and $\mathrm{T} 1$; on the contrary, $\mathrm{MET}_{\max }$ of the elderly group no significant improvement between T0 and T1. However, the exercise capacity of all groups showed improvement between T0 and T2. Moreover, the mean $\mathrm{MET}_{\text {max }}$ of younger patients after 6 weeks of CR improved $6.08 \pm 0.75$ to $7.28 \pm 1.81$ compared to baseline, while the elderly patients only improved $5.18 \pm 1.32$ to $5.40 \pm 1.29$. However, after 12 weeks of $C R$, the mean $\mathrm{MET}_{\text {max }}$ of younger patients was improved $6.08 \pm 0.75$ to $7.52 \pm 1.66$ and the mean MET $_{\max }$ of elderly patients improved $5.18 \pm 1.32$ to $6.29 \pm 2.00$ compared to baseline.

Our reported $\mathrm{MET}_{\max }$ was smaller than that reported in previous studies. This result may be due to differences in patient groups. Previous study groups included the entire group regardless of risk factors. Our patient group excluded patients with low risk factors on the risk classification. Moreover, the total $\mathrm{MET}_{\max }$ was small because the experiment was only performed with patients with a $\mathrm{MET}_{\max }$ value of less than 7.

As in previous studies, our study revealed that CR is effective in elderly patients. Our study showed that the amount of recovery in the elderly group was relatively low compared to previous studies. This could be interpreted as a difference in the patient groups due to excluding patients with low risk factors as stated above.

The systematic literature review by Strid et al. [5] showed that long duration of CR could lead to higher physical activity and exercise. However, the exact duration of CR to improve exercise capacity in elderly patients has not been specifically discussed. 
ETT at 6 and 12 weeks after CR confirmed the improvement in exercise capacity in the elderly and younger groups. There was a significant increase in exercise capacity after 12 weeks in elderly and younger patients, but no significant improvement in exercise capacity was observed after CR for 6 weeks in elderly patients.

The initial values of the two groups were different, so we checked the changing ratio for correction (Fig. 1). The changing ratio of $\mathrm{MET}_{\max }$ in the younger patient group showed that the degree of recovery is mainly in the first 6 weeks, and the changing ratio of the elderly patient group showed a steady recovery in 12 weeks.

Our results suggest that the elderly patients should have a longer phase II CR duration than the younger ones to improve their exercise capacity. The duration of phase II CR in Europe is reported to average 8 weeks (3-14 weeks); when outpatient rehabilitation was performed the duration of phase II programs ranged from 7 to 26 weeks [10]. However, this study could be interpreted to demonstrate the duration of phase II programs in elderly AMI patients requires at least 6 weeks. Furthermore, we suggest that continuous aerobic exercise is needed during this period.

There are some limitations to our study. First, our study was a retrospective review. Therefore, the results have been influenced by the nature of observation. This study excluded patients with low risk factors to clarify the duration of phase II CR for patients with moderate to severe risk factors. Second, the sample size was relatively small. This could be influenced by patients' motivation, general conditions, and the cost of follow-up ETTs. Finally, our study was performed without distinction between center-based and home-based CR. We allowed patients to choose between center-based or home-based CR. Because of this, the exercise intensity, timing, or duration for each patient may not have been strictly controlled. In the case of the home-based CR, we used patients' self-exercise diaries, phone calls, or exercise history recordings. The actual adherence cannot exclude the bias that arises from dependence on the patient's memory or diary. It makes it difficult to quantify the CR program.

In future studies, it is necessary to determine the specific time of improvement by subdividing the follow-up timing. Additionally, quantification of exercise intensity and frequency of participation is needed. Furthermore, the addition of other types of exercise (strengthening or balance exercise) is worth considering.
In conclusion, AMI patients aged 65 years or older show improvement 12 weeks after completion of the first ETT. However, patients under 65 years of age saw improvement 6 weeks after completion of the first ETT, while patients over 65 took more than 6 weeks to show improvement. Therefore, people aged 65 years or older should be encouraged to participate in CR for a sufficient period of time (6 weeks or more).

\section{CONFLICT OF INTEREST}

No potential conflict of interest relevant to this article was reported.

\section{ACKNOWLEDGMENTS}

This work was supported by the National Research Foundation of Korea (NRF) grant funded by the Korean government (MSIP) (2019R1I1A3A01060059).

\section{AUTHOR CONTRIBUTION}

Conceptualization: Han JY. Methodology: Kim KH, Jang YC. Formal analysis: Park HK, Song MK. Funding acquisition: Han JY. Project administration: Han JY. Writing original draft: Kim KH. Writing - review and editing: Choi IS, Song MK, Han JY. Approval of final manuscript: all authors.

\section{REFERENCES}

1. McConnell TR, Laubach CA 3rd, Szmedra L. Age and gender related trends in body composition, lipids, and exercise capacity during cardiac rehabilitation. Am J Geriatr Cardiol 1997;6:37-45.

2. Lavie CJ, Milani RV, Littman AB. Benefits of cardiac rehabilitation and exercise training in secondary coronary prevention in the elderly. J Am Coll Cardiol 1993;22:678-83.

3. Anderson L, Oldridge N, Thompson DR, Zwisler AD, Rees K, Martin N, et al. Exercise-based cardiac rehabilitation for coronary heart disease: Cochrane systematic review and meta-analysis. J Am Coll Cardiol 2016;67:1-12.

4. Lavie CJ, Milani RV. Effects of cardiac rehabilitation programs on exercise capacity, coronary risk factors, 
behavioral characteristics, and quality of life in a large elderly cohort. Am J Cardiol 1995;76:177-9.

5. Strid C, Lingfors H, Fridlund B, Martensson J. Lifestyle changes in coronary heart disease: effects of cardiac rehabilitation programs with focus on intensity, duration and content: a systematic review. Open J Nurs 2012;2:420-30.

6. Baldasseroni S, Pratesi A, Francini S, Pallante R, Barucci $\mathrm{R}$, Orso $\mathrm{F}$, et al. Cardiac rehabilitation in very old adults: effect of baseline functional capacity on treatment effectiveness. J Am Geriatr Soc 2016;64:1640-5.

7. Bartels M, Prince DZ. Acute medical conditions. In: Cifu DX, editor. Braddom's physical medicine \& rehabilitation. 5th ed. Philadelphia: Elsevier; 2016. p. 57196.

8. Santiago de Araujo Pio C, Marzolini S, Pakosh M, Grace SL. Effect of cardiac rehabilitation dose on mortality and morbidity: a systematic review and metaregression analysis. Mayo Clin Proc 2017;92:1644-59.

9. Price KJ, Gordon BA, Bird SR, Benson AC. A review of guidelines for cardiac rehabilitation exercise programmes: Is there an international consensus? Eur J Prev Cardiol 2016;23:1715-33.

10. Vanhees L, Martens M, Beloka S, Stevens A, Avram A, Gaita D. Cardiac rehabilitation: Europe. In: Perk J, et al., editors. Cardiovascular prevention and rehabilitation. London: Springer; 2007. p. 30-3.

11. Kraal JJ, Vromen T, Spee R, Kemps HMC, Peek N. The influence of training characteristics on the effect of exercise training in patients with coronary artery disease: systematic review and meta-regression analysis. Int J Cardiol 2017;245:52-8.

12. Lavie CJ, Milani RV. Disparate effects of improving aerobic exercise capacity and quality of life after cardiac rehabilitation in young and elderly coronary patients. J Cardiopulm Rehabil 2000;20:235-40.

13. Menezes AR, Lavie CJ, Forman DE, Arena R, Milani RV, Franklin BA. Cardiac rehabilitation in the elderly. Prog Cardiovasc Dis 2014;57:152-9.

14. Pasquali SK, Alexander KP, Peterson ED. Cardiac rehabilitation in the elderly. Am Heart J 2001;142:74855.

15. Cannistra LB, Balady GJ, O'Malley CJ, Weiner DA, Ryan TJ. Comparison of the clinical profile and outcome of women and men in cardiac rehabilitation. Am J Cardiol 1992;69:1274-9.

16. Anderson L, Sharp GA, Norton RJ, Dalal H, Dean SG, Jolly K, et al. Home-based versus centre-based cardiac rehabilitation. Cochrane Database Syst Rev 2017;6:CD007130. 